\title{
Analyzing Performance of a Global Help Desk Team Operation - Country Handoffs, Efficiencies and Costs
}

\author{
Akhil Kumar \\ Smeal College of Business \\ Penn State University \\ University Park, Pa 16802. USA \\ akhil@psu.edu
}

\begin{abstract}
In this paper, we study the characteristics of a global help desk operation using Volvo IT support help desk. We analyze the incidents log produced by the Volvo IT department that gives the full path of an incident and the participating country and worker at each step. Our main goal is to gain a better understanding of the nature of international traffic flows that occur during the resolution of incidents. We find that increasing the number of participating countries negatively affects IT service efficiency metrics. Further, we breakdown international traffic flows (by highand low-efficiency countries) and examine the country effects and their implications in terms of efficiency and cost in considerable detail. The results and insights gained are discussed at length and can help in optimizing incident resolution workflows from a cost, efficiency and resource allocation perspective.
\end{abstract}

\section{Introduction}

In a global IT support operation such as a help desk to resolve software incidents, several support technicians, specialists and other staff collaborate to solve problems that are reported on help tickets. Some issues are resolved in the country of origin, while others may be routed to the help desk in another country where greater expertise is available, or there is more spare capacity. In such an operation a series of handoffs between task actors (or workers) are involved who perform successive tasks on the incident or process instance such as recording, dispatch, resolution, testing and closure, until the problem is resolved. After an actor completes her task she hands off the process instance or case to the next actor. Such behavior is observed in various kinds of application areas ranging from medicine and software development to insurance claim processing. Some handoffs also occur in a ping-pong pattern such that an actor A hands off a task to actor B only to have it returned later, either after some work is done or

\author{
Siyuan Liu \\ Smeal College of Business \\ Penn State University \\ University Park, Pa 16802. USA \\ sx168@psu.edu
}

just untouched. This leads to an actor handoff pattern represented by the sequence A-B-A, A-B-C-D-A, etc. A group of workers collaborating to resolve an incident in this manner forms a team, which is international if workers from different countries are involved.

By applying a process mining algorithm [21] we may discover a process model or a typical workflow that always starts with, say, task T1; next, tasks T2 and T3 appear in a choice (or alternative) structure and are followed by a parallel structure of T4 and T5 that can appear in any order. A drawback with most process mining approaches though is that when considerable variety is inherently present in a process, capturing it in precise detail leads to a "spaghetti-like" model that is unreadable. A help desk process is an example process where there is considerable variety because decisions must be made about the next step or handoff through human agency on a rather ad hoc basis depending often upon their tacit knowledge of several factors like the suitability of other workers, their availability, etc. Often an incident is misrouted multiple times by being repeatedly assigned to the wrong worker or queued up in the worklist of a worker. Our goal is to understand the nature of work handoff patterns across countries that arise in such a setting.

Institutions "are embodied in routines or organizing schemas" for learning, problem solving and normal activities [19]. Institutions (formal and informal) shape the cultural identify of an individual [7]. To the extent they differ across nationalities they create room for friction and negative team dynamics, and at the same time also give rise to different perspectives. Trust and rapport develop within such teams over time [5]. The organizational management literature has identified the role of nationality as it affects performance of individuals in multinational teams over time [22]. Much of this research is in the context of teams at various management and executive levels, while help desk operations have not been studied from this angle.

Our work also fits into the broader area of work distribution and resource allocation studied in business 
process management (e.g. [4],[8],[11],[12],[16]), but our main focus lies in patterns of handoffs across countries. To enable our work, we use an IT support dataset from an international company with global operations. Our main research questions (RQ) are as follows:

RQ1: How does performance for incidents that are resolved collaboratively by workers from multiple countries compare with those that are resolved in a single country?

RQ2: Are their pairs of countries that are significantly different in terms of their productivity metrics?

RQ3: What is the nature of incident traffic flows among countries?

The common thread running through the RQs is that of traffic flows across countries. The nature of traffic flows (RQ3) is influenced by inter-country productivity differences (RQ2) and collaboration dynamics (RQ1) across countries. Our study was made possible by access to a large log from the incident resolution process at the Volvo automotive company [3]. This data set is public and hence the results can be verified. This data set has been analyzed extensively from a process mining perspective to extract the underlying process models and the kinds of behaviors they exhibit (see for instance, [1]). However, the international aspect in the resolution of incidents has not received any attention. Our goal is to revisit the dataset from this new perspective to understand the nature of country handoff patterns that arise when incidents are resolved globally since $56.4 \%$ of incidents are resolved in more than one country.

This paper is organized as follows. After reviewing literature and theory briefly in Section 2, Section 3 gives some case background and presents a preliminary overview of the data. Then, Section 4 describes our log data and the main results of our analysis. Next, Section 5 applies the Wilcoxon test to check if differences between countries are significant and also analyzes traffic flows across countries. Later, Section 6 offers a discussion of our findings and the limitations of our work. Section 7 shares some concluding thoughts along with suggestions for future work.

\section{Related work and theory}

Cooperative teams play a large role in the success of any endeavor. In todays global corporation, activities are frequently carried out by multinational teams. There is already considerable research on the implications of cultural diversity on work efficiency and team effectiveness in terms of age [10], gender [13],[23] and ethnicity [3], while very little empirical work has directly addressed the importance of nationality diversity. Though the implications of nationality may be profound, however, the research on relationship between nationality diversity and work performance remains inconsistent and inconclusive.

Some researchers found a positive link between nationality diversity and work performance, while others did not. Watson, et al. [22] showed that towards the end of their 17-week longitudinal study, the culturally diverse groups had become more effective on tasks like identifying problem perspectives and generating solution alternatives than a culturally homogeneous group. Hambrick et al. [9] observed that nationality will shape individuals' behavior when facing task stimuli through an individuals distinct combination of values, cognitive schema, demeanor, and language. At the same time, multinational team members may struggle in integrating their diversified experiences, and in generating creative ideas, to produce better performance when dealing with complex tasks.

Social categorization theory explains how people place themselves in social categories and its effects on group dynamics. Drawing on this theory, Chatman and Flynn [5] found that demographic heterogeneity among team members delayed the formation of cooperative norms among them, but this effect dissipated with time. It has also been found that nationality and culture determine communication patterns and interaction styles [7]. Nielsen and Nielsen [14] find that nationality diversity has positive implications for performance and longer tenured terms, and highly internationalized firms and munificent environments will further consolidate this positive effect. Still, Zoogah et al. [23] found that the direct relationship between nationality diversity and work effectiveness was not significant and hypothesized two possible explanations for it to be mistrust and withholding effect. Another recent study [17] points to institutional and societal factors as having high driving power in team work noting that such factors are strongly affected by and tied to the national culture.

Much work has been done on allocation of tasks to workers in a business process management (BPM) context from multiple perspectives such as: performance and quality tradeoffs [11]; priorities [4]; risk [6]; and abilities [8]. A taxonomy that integrates many dimensions of resource allocation is developed by Arias, et al. [2]. The thrust of this line of research is to find the best or "optimal" assignments of tasks in a process instance to workers by taking one or more of these criteria into account using algorithms based on heuristics and rules, or discrete optimization methods. However, nationality has not been considered as a criterion for work allocation. In 
other related work, many workflow resource patterns have been developed to offer tasks to workers through pull and push mechanisms [16]. An organization such as Volvo can use one or more of these patterns to assign work and to help workers manage their work queues efficiently. Finally, work in organizational mining is relevant as it helps to understand roles performed by workers and to discover organizational relationships [20]. Such information can also be used to extract team structure [18]. Social network-based work allocation mechanisms that make use of the social graph of interactions among workers for making assignments have been developed in [12].

\section{Preliminary background}

\subsection{Case specifics}

Our analysis is based on a publicly available dataset that was released as a part of the BPI Challenge in 2013 [3]. The dataset spans from March 2010 to May 2012, and is a $\log$ of reported incidents or cases. Each incident has a unique serial or SR number. After a record is created for an incident, it passes through multiple workers or owners in a support team as they try to identify the cause of the problem and find a resolution for it. Thus, there are many log records for each incident to reflect any status or owner change until the problem is resolved. A log record captures the status of the incident and includes information like serial number, date-timestamp, status, sub-status, (business) impact, product, country, owner, support team, and organizational line as shown in the partial log of Table 1. There are 7,554 cases or incidents and 65,553 events in the $\log$ for an average of 8.7 events per incident.

The owner attribute in the log record denotes the actual actor who performs a task. When two successive (in time sequence) $\log$ records with the same SR Number have different owners, then it indicates a handoff of work from the previous owner to the new one. The dataset was loaded in a MySQL database for the analysis. We first removed the log records for the owner 'Seibel' because this is the information system, and not a human owner. Our focus was on studying the effect of handoffs among human actors only since a resource allocation algorithm can only select a specific human from a set of alternatives. Then we removed all records for incidents where only one human owner was involved and for incidents that were not resolved or completed. This left us with 4375 incidents - 1755 low impact, 2413 medium impact, 204 high impact and 3 major impact incidents. Here impact is interpreted to mean the business effect that this incident is likely to have on the operations of the firm. A break-down of incidents by impact level and number of countries involved in their resolution appears in Table 2. In our further analysis, we have neglected the 3 major impact incidents because they are statistically insignificant. After removing duplicate log records there were 46,323 event $\log$ entries in the database for the remaining 4,372 incidents. Table 3 shows a breakdown of the events by status. Notice how $22 \%$ of the events are for a status of 'queued' awaiting assignment, while another $12.33 \%$ events record a sub-status of 'wait'.

Our methodology in this paper combines use of SQL queries in MySQL and Excel; statistical testing to determine significance of trends; and visualization to present our findings. Several queries were written in MySQL using constructs like JOIN, GROUP BY, ORDER BY, COUNT, CONCAT, etc. to categorize the help tickets by status, extract help ticket country patterns, calculate metric values and determine traffic flows. Results from MySQL were further analyzed in Excel. Statistical tests were performed to check for normality (Jarque-Bera) and compare samples (Kruskal-Wallis). Several linear regressions were performed to understand significance of relationships between variables, and the Wilcoxon rank test was used to test if efficiency was significantly different across countries. Visualizations like box plots were used to gain deeper insights into the data.

We first ran the Jarque-Bera test to check the normality of duration (i.e. the elapsed time from start to finish) of incidents with three different impact values, and the p-value was 0.0000 , showing that elapsed time of an incident in each impact type is not normally distributed. Hence, the non-parametric Kruskal-Wallis test was applied to check whether the three types of incidents originate from the same distribution, and the p-value was 0.0001, which shows there is significant difference in the duration of an incident at different impact values.

For a more detailed test, we paired the values of impact, using Wilcoxon rank-sum test (also known as the Mann Whitney U Test). It tests for differences between two groups on a single, ordinal variable with no specific distribution [15] by comparing if a randomly selected value from one sample will be less (or greater) than a randomly selected value from a second sample. It was found that low impact incidents are significantly different (of longer duration) from the medium and high impact incidents on duration, but medium and high impact incidents' duration is not significantly different. 
Table 1. A snapshot of the incident log

\begin{tabular}{|c|c|c|c|c|c|c|c|}
\hline SR Number & Date & Status & SubStatus & Impact & Product & Country & Owner Name \\
\hline $1-503573772$ & $2011-02-24$ & Accepted & In Progress & Medium & PROD706 & Belgium & Bert \\
\hline $1-503573772$ & $2011-02-24$ & Queued & Not Assigned & Medium & PROD706 & Belgium & Bert \\
\hline $1-503573772$ & $2011-02-28$ & Accepted & In Progress & Medium & PROD706 & Netherlands & Luc \\
\hline $1-503573772$ & $2011-02-28$ & Accepted & In Progress & Medium & PROD706 & Belgium & Henk \\
\hline $1-503573772$ & $2011-02-28$ & Queued & Not Assigned & Medium & PROD706 & Belgium & Henk \\
\hline $1-503573772$ & $2011-02-28$ & Queued & Not Assigned & Medium & PROD706 & Netherlands & Luc \\
\hline
\end{tabular}

Table 2. Incidents by impact level and number of countries involved in resolution

\begin{tabular}{|c|c|c|c|c|c|c|c|}
\hline & 1 & 2 & 3 & 4 & 5 & 6 & Total \\
\hline Low & 575 & 827 & 293 & 56 & 4 & 0 & 1755 \\
\hline Med. & 1260 & 766 & 274 & 88 & 22 & 3 & 2413 \\
\hline High & 72 & 97 & 25 & 8 & 2 & 0 & 204 \\
\hline Major & 0 & 1 & 2 & 0 & 0 & 0 & 3 \\
\hline Total & 1907 & 1691 & 594 & 152 & 28 & 3 & 4375 \\
\hline
\end{tabular}

Table 3. A breakup of 46323 incidents by status

\begin{tabular}{|l|l|l|l|}
\hline Status & Sub-status & Count & $\%$ \\
\hline \multirow{5}{*}{ Accepted } & Assigned & 3,006 & $6.49 \%$ \\
\cline { 2 - 4 } & In progress & 17,943 & $38.73 \%$ \\
\cline { 2 - 4 } & Wait, & 5,713 & $12.33 \%$ \\
& $\begin{array}{l}\text { Wait-Customer, } \\
\text { Wait-User, } \\
\text { Wait-Implemented, } \\
\text { Wait-Vendor }\end{array}$ & & \\
\hline Completed & Closed, Resolved & 9,349 & $20.18 \%$ \\
\hline Queued & $\begin{array}{l}\text { Awaiting } \\
\text { Assignment }\end{array}$ & 10,307 & $22.25 \%$ \\
\hline Unmatched & Unmatched & 15 & $0 \%$ \\
\hline
\end{tabular}

\subsection{International content and country handoff patterns}

Figure 1 shows a breakdown of domestic and international content in the incident resolution process from the Top- 8 countries in terms of incidents. An incident is resolved domestically if all the events are performed by workers in its country of origin. There were 1,907 incidents out of 4,375 (i.e. $43.6 \%$ ) that were handled domestically, while the remaining $56.4 \%$ incidents were "international," i.e. they involved handoffs across countries. Next, we look at the Top-15 country handoff patterns for the international incidents in Table 4 by frequency. A breakup of the incidents by impact level is also given. This table shows that more low impact incidents are resolved internationally than medium impact ones even though there are more medium impact incidents in the dataset. In fact, many low impact incidents are sent from low wage countries like India, Brazil and Poland to high wage countries like Sweden for resolution. We will return to this point in Section 5 .

\section{Evaluation of international incidents}

We first propose several metrics that may be used in evaluation. These were chosen because they are typical efficiency and productivity metrics used for evaluation:

Metric0 $(\mathrm{M} 0)=$ Average $($ Avg.) operations cost or Avg. \# of Events to resolve an incident

Metric1 $(\mathrm{M} 1)=$ Avg. \# of Events/Worker

Metric2 $($ M2) $=$ Avg. Ticket Duration/Worker

Metric3 (M3) = Avg. \# of Events/Handoff

Metric4 (M4) = Avg. Ticket Duration/ Handoff

M0 is a measure of operations cost, while M1-M4 are measures of efficiency of incident resolution. Informally, an event represents a step in the incident resolution process and each step incurs an additional cost involved in resolution. Table 5 shows the statistics for these four metrics for incidents where from 1 to 6 countries are involved.

We find that when the number of countries increases, then M1 and M2 increase, while M3 and M4 drop. This means that more events happen per worker and the time spent by each worker also increases with more countries. However, M3 and M4 decrease as the number of countries increases. This means that handoffs are frequent, but the same workers are involved in the incident multiple times, thus suggesting that when more countries are involved it is normal for work to return to the home country for closure. We see this trend in many of the country handoff patterns shown in Table 4.

A regression was performed to derive M3 or Events/Handoff in terms of workers, countries and impact level. The results in Figure 2 show that all three independent variables are significant. Further, observe that the coefficients for Countries and Workers are negative. As noted above this is because handoffs are more frequent with increasing number of countries, but the total number of handoffs also increases as stated because the incident returns to the same workers who were involved with it previously. Hence, such handoffs 


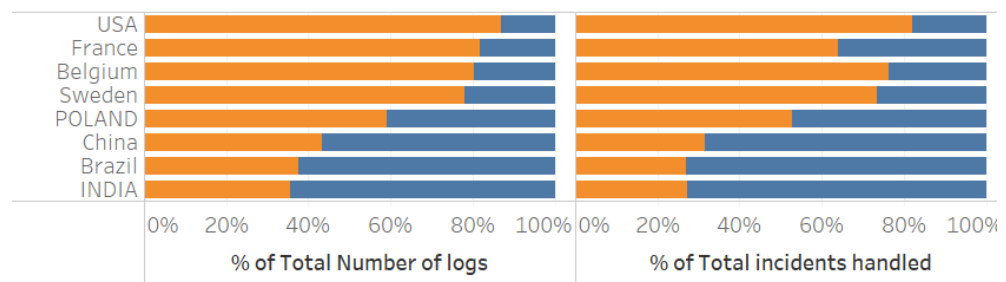

Figure 1. Mix of domestic (yellow) and international (blue) content event counts

Table 4. Most frequent country handoff patterns by impact frequency

\begin{tabular}{|l|l|l|l|l|}
\hline Pattern & Low & Med. & High & Total \\
\hline Sweden-Poland & 85 & 46 & 3 & 134 \\
\hline Poland-Sweden & 90 & 32 & 1 & 123 \\
\hline $\begin{array}{l}\text { Sweden-Poland- } \\
\text { Sweden }\end{array}$ & 15 & 67 & 1 & 83 \\
\hline $\begin{array}{l}\text { Poland-Sweden- } \\
\text { Poland }\end{array}$ & 44 & 14 & 0 & 58 \\
\hline India-Sweden & 33 & 15 & 5 & 53 \\
\hline India-Poland & 32 & 13 & 14 & 49 \\
\hline Poland-India & 33 & 15 & 1 & 49 \\
\hline Poland-France & 41 & 2 & 0 & 43 \\
\hline $\begin{array}{l}\text { Belgium-France- } \\
\text { Belgium }\end{array}$ & 0 & 41 & 0 & 41 \\
\hline Sweden-India & 10 & 23 & 4 & 37 \\
\hline France-Poland & 29 & 8 & 0 & 37 \\
\hline $\begin{array}{l}\text { Sweden-India- } \\
\text { Sweden }\end{array}$ & 8 & 16 & 12 & 37 \\
\hline Brazil-Sweden & 26 & 7 & 3 & 36 \\
\hline $\begin{array}{l}\text { Sweden-Brazil- } \\
\text { Sweden }\end{array}$ & 2 & 27 & 1 & 30 \\
\hline $\begin{array}{l}\text { India-Sweden- } \\
\text { India }\end{array}$ & 23 & 4 & 1 & 28 \\
\hline
\end{tabular}

are wasteful and inefficient. They are also a sign of misrouting of the incident. In addition, the coefficient of the LowImpact dummy variable shows that there are more events for low impact incidents than for high or medium ones. The negative coefficient for number of workers indicates that as there are more workers each one is performing fewer events.

Figure 3 gives a plot of operations cost (\#events) vs. \#workers where the three curves correspond to low (1-2), medium (3-4) and high (5+) number of countries. This shows that as the number of countries increases, there is a corresponding increase both in the number of workers as also in event logs. Thus, this further corroborates the statistics in Table 5 and suggests that it is inefficient to involve a large number of countries in incident resolution. Moreover, Figure 4 gives a box plot showing the number of events held in a queue (with a status showing "queued") as a percentage of the total number of events for incidents involving a range of countries from 1 to 6 . This shows that with more countries the queues build up for processing of a multi-country incident. Hence, this further supports the statistics in Table 5, and shows that it is inefficient to involve many countries in incident resolution.

\section{Inter-country traffic flows}

\subsection{Efficiency across countries}

Now, we investigate the kinds of differences that arise across countries by examining the performance of workers. Figure 5 shows a plot of the number of events (or log records) vs. number of incidents in the Top-8 countries. In this plot, each worker (or incident owner) is represented by a dot. A regression line is also drawn through the worker dots for each country. The slope of this line is a measure of metric M1. A higher slope in this figure corresponds to lower efficiency. It is evident that Belgium has the highest slope that corresponds to the weakest performance, while Sweden has the least slope that corresponds to the strongest performance. The other countries fall in between. Further, the slopes of China, India, Poland and Brazil are all larger than those of USA, France and Sweden. We also ran the Wilcoxon Rank test to compare certain pairs of countries, e.g. Sweden with Poland on Duration and operations cost. On both the metrics, the null hypothesis that the two countries were equal was rejected at the $\mathrm{p}=$ 0 level.

\subsection{Traffic patterns across countries}

Next, we study the traffic across countries to better understand the nature of such flows. To determine these patterns, we looked at the "Owner Country" field for all events that were performed on an incident. Since we also know the owner country of an incident (i.e. where it originated), if an event is produced in another country than the origin country it represents a transfer of work 


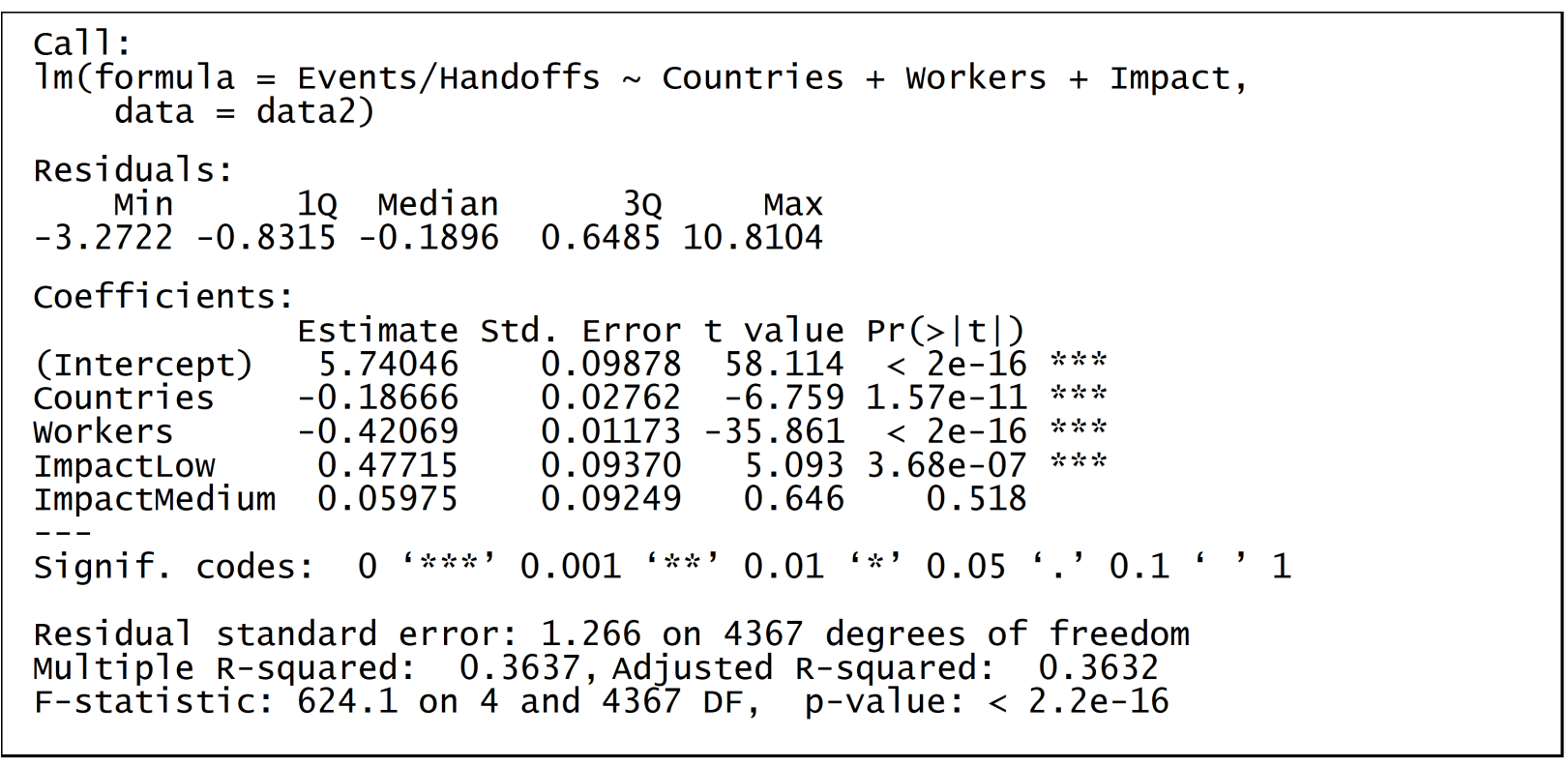

Figure 2. Regression to determine M3 (Events/Handoff)

Table 5. Summary evaluation on four metrics

\begin{tabular}{|c|c|c|c|c|c|c|c|c|c|}
\hline $\begin{array}{c}\text { Count- } \\
\text { ries }\end{array}$ & Count & \multicolumn{5}{|c|}{ Basic Data } & \multicolumn{4}{c|}{ Metrics } \\
\hline & & Events & Duration & Workers & $\begin{array}{c}\text { Hand- } \\
\text { offs }\end{array}$ & $\begin{array}{c}\text { Events/ } \\
\text { Worker }\end{array}$ & $\begin{array}{c}\text { Duration/ } \\
\text { Worker }\end{array}$ & $\begin{array}{c}\text { Events/ } \\
\text { Handoff }\end{array}$ & $\begin{array}{c}\text { Duration/ } \\
\text { Handoff }\end{array}$ \\
\hline 1 & 1907 & 7.91 & 14.34 & 2.75 & 2.00 & 2.91 & 5.36 & 4.70 & 8.96 \\
\hline 2 & 1691 & 10.11 & 21.40 & 3.44 & 3.08 & 2.93 & 6.31 & 4.02 & 8.43 \\
\hline 3 & 594 & 16.56 & 22.79 & 5.55 & 5.99 & 2.97 & 4.39 & 3.00 & 4.58 \\
\hline 4 & 152 & 20.58 & 21.84 & 6.81 & 7.95 & 3.00 & 3.16 & 2.71 & 3.10 \\
\hline 5 & 28 & 37.25 & 37.93 & 9.75 & 15.39 & 3.58 & 3.78 & 2.51 & 2.76 \\
\hline 6 & 3 & 39.33 & 29.33 & 11.33 & 20.33 & 3.52 & 2.44 & 1.95 & 1.36 \\
\hline
\end{tabular}

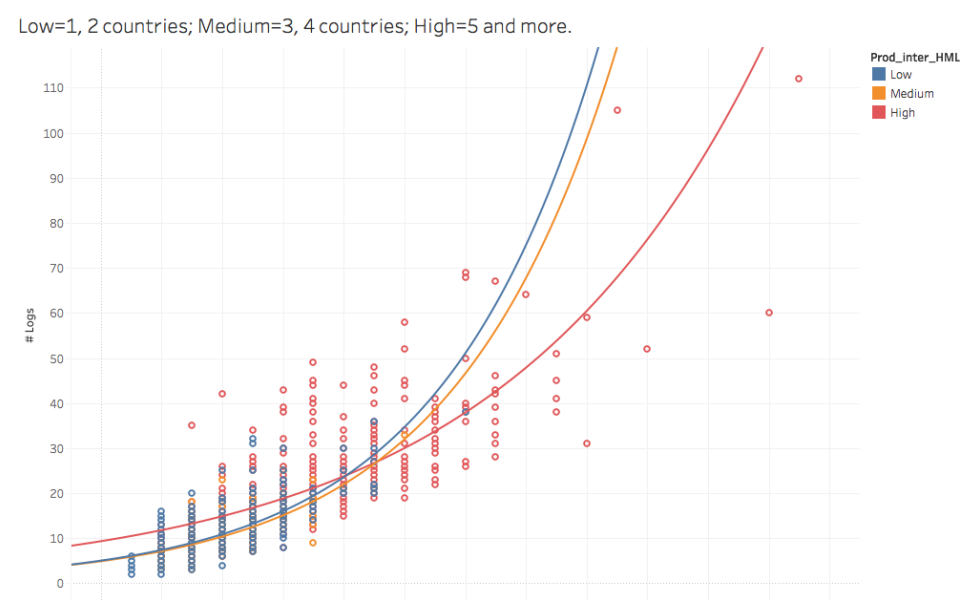

Figure 3. Operations cost vs. \#Workers across three country groups (each dot is an incident) 
Waiting time over the number of

countries on incidents

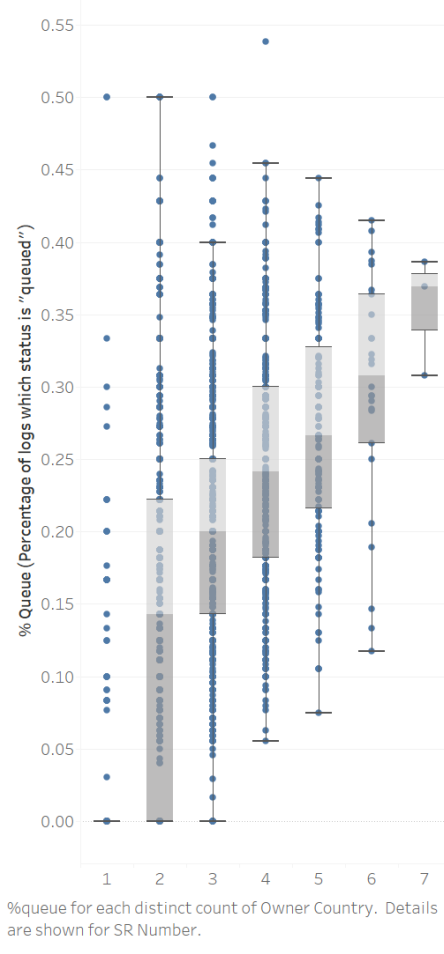

Figure 4. Percentage of event log records with "queued" status vs. \# of countries

from the country of origin to the foreign country. In this table (see Table 6), the columns show the outgoing traffic from each country and the rows correspond to the incoming traffic into each country. The last row and column give the total values. The diagonal entries are the largest for each country as they represent the volume of work performed internally.

Now, based on the efficiencies in Figure 5, we can divide the 8 countries in Table 6 into two groups of "low-" and "high-efficiency" countries, where Belgium, Brazil, China, India and Poland are in the former group, while France, Sweden and USA are in the latter. Hence, by condensing Table 6 we obtain Table 7.This table also breaks down the totals across inter-country traffic flows by percentage within these groups. We observe that a full $80 \%$ of the total traffic flow stays with the respective low- and high-efficiency groups. Of the remaining traffic, $11 \%$ flows from high- to low-efficiency countries and $9 \%$ in the reverse direction. Another way to interpret the results is that low-efficiency countries send $19.4 \%$ of their out-traffic to high-efficiency ones, while $20.7 \%$ traffic flows in the reverse direction.

Thus far, our discussion has hinged entirely on the numbers of events in the log. Next, we consider the effect of introducing costs into the picture given that the cost of workers varies from country to country and some countries have a wage advantage

\subsection{Economic cost considerations}

Above the operations costs are measured in terms of number of events. The next step in our analysis is to consider the operations cost from a cost perspective. Accordingly, since wages for the same job, say a programmer or a technical support specialist, vary between countries it would be useful to incorporate the effect of that factor into our analysis. As a proxy for wages (since wages vary widely across classes of IT support workers that are involved in incident resolution and also across cities within the same country), we decided to use the costs of living index of different countries in our study to represent the differential wages of workers across various countries. The cost of living index for the eight main countries in our study is shown in Table 8.

Next, in Table 9, we summarize a variety of information, such as \# of workers in the support team of that country, the total \# of incidents originating in that country and the number that is resolved domestically for these eight countries. Then, we also derive the percentage of incidents that are handled entirely domestically along with incidents per worker. In addition, the table shows the number of events per incident for incidents processed fully within a country as a measure of the operational cost incurred in that country. This metric varies from a low of 6.68 for USA (most efficient) to a high of 10.46 for Poland (least efficient). Recall that this measure is determined for the special case when an incident is processed entirely within the same country and so this result differs from Figure 5 where some contribution of workers from other countries could be mixed up across the incidents. When this number is multiplied by the cost of living index we get a measure of the relative monetary cost of processing an instance in that country. Note that these numbers are not in any actual currency rather they are a measure of the relative costs in an arbitrary currency. These costs vary from 227 in India to 676 in France, i.e. by a factor of 3. The other countries fall within this range, with Poland, China and Brazil being closer to India ("low-cost" group), while Sweden and USA are closer to France ("high-cost" group).

On further examination of Table 9, we observe that the first four countries in this table fall in the so-called "low-cost" group with cost in the 200-400 range, while the last four countries in the "high-cost" group have costs in the 575-675 range. Notably, 


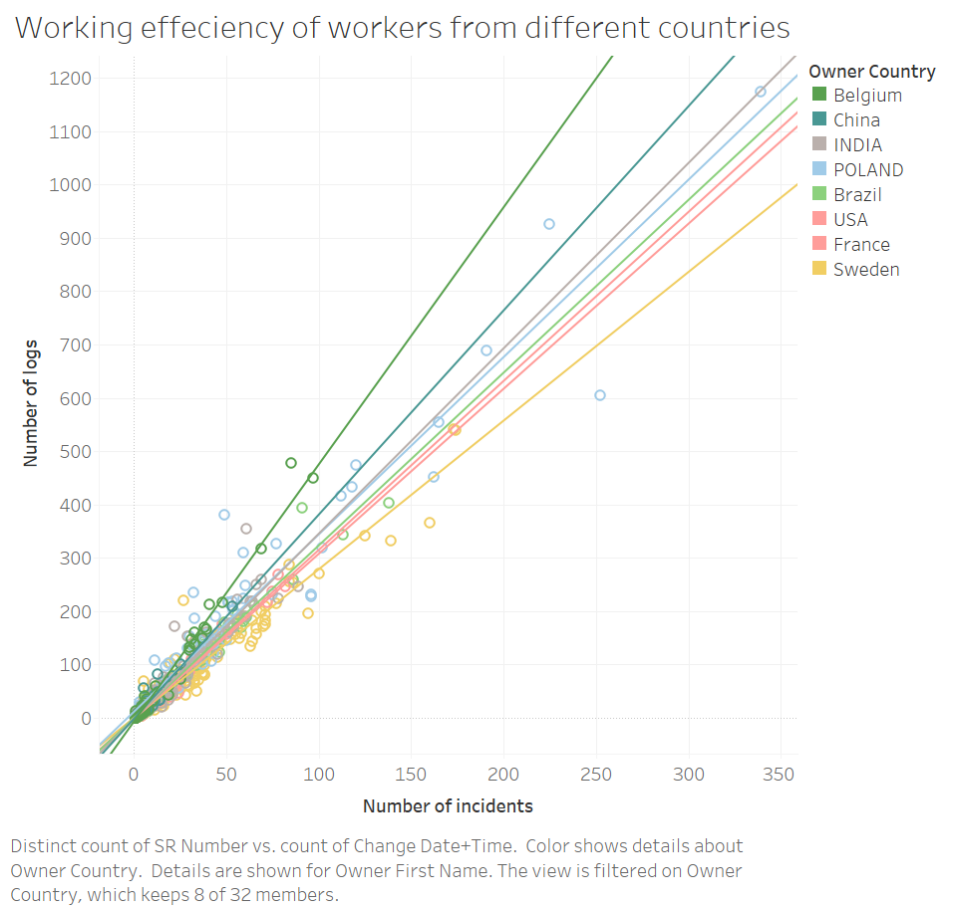

Figure 5. Working efficiency of workers from different countries $($ dot $=$ worker $)$

India and Poland in the low-cost group handle a small percentage (approximately 22\%) of their own incidents domestically, while Sweden and USA in the high-cost group handle a large percentage (approximately $61 \%$ ) of their incidents domestically. Besides, both Sweden and USA also have higher incidents per worker ratios than India.

On comparing Tables 6 and 9, notice that Sweden with a cost per incident of 614 is handling about $33 \%$ of the total work in terms of events, while India with a cost of 227 is handling only about $15 \%$ of the work. This suggests that: (1) low cost countries could handle more of their incidents locally; and (2) more work can be shifted from high- countries to low-cost ones. Clearly, there is need for work allocation mechanisms to enable this behavior. Of course, one must also consider that there is a higher level of skills in advanced countries like Sweden and US than in low-cost ones.

\section{Discussion and limitations}

This analysis helps us, most importantly, to understand the traffic flow patterns (RQ3) among individual countries, and particularly, among low- and high-efficiency countries (RQ2). These two groups were identified based on differences in incident resolution duration and operations cost. We found that $80 \%$ of the total traffic flow stayed within each of these two groups.
The flows of the inter-group traffic were divided about equally between the two groups. The traffic flows from the high- to low- efficiency group can be explained by the analysis of economic considerations (see Table 9) that suggests that incident resolution cost per incident is lower in the low-efficiency group. A possible explanation for the flows in the reverse direction is based on skill levels. Thus, one would expect that skill levels are higher in high-efficiency countries leading to the latter (low to high) flows when certain specialized skills are called for. In particular, the highest volume of inward flow $(12,926$ events, or $33.9 \%$ of the total flow) takes place to Sweden which is the headquarters of Volvo and the likely repository of the highest skilled resources in the firm. Further, the second highest inflow (8,734 events, or $22.9 \%$ of the total flow) takes place to Poland that has the second least cost per incident from Table 9. Sweden and Poland are also the two countries that participate the most in inter-country incidents as shown in Table 4.

In addition, our analysis shows that when more countries collaborate in incident resolution, the number of $\log$ events and number of workers are both higher (see Figure 3). Moreover, the number of queued incidents also goes up (see Figure 4). This is a sign that collaboration across countries (RQ1) is fraught with pitfalls. First, there can be language and other communication barriers across countries in terms of 
Table 6. Traffic flows across countries in terms of numbers of events

\begin{tabular}{|c|c|c|c|c|c|c|c|c|c|}
\hline & Belgium & Brazil & China & France & India & Poland & Sweden & USA & Total -In \\
\hline Belgium & 2125 & 0 & 0 & 0 & 55 & 76 & 95 & 6 & 2357 \\
\hline Brazil & 325 & 1650 & 2 & 58 & 309 & 1170 & 401 & 158 & 4073 \\
\hline China & 38 & 0 & 392 & 1 & 37 & 94 & 79 & 22 & 663 \\
\hline France & 101 & 0 & 0 & 1170 & 45 & 53 & 66 & 17 & 1452 \\
\hline India & 356 & 26 & 344 & 100 & 2393 & 1438 & 668 & 402 & 5727 \\
\hline Poland & 563 & 36 & 45 & 554 & 918 & 4977 & 1537 & 104 & 8734 \\
\hline Sweden & 409 & 14 & 20 & 310 & 1387 & 1209 & 9355 & 222 & 12926 \\
\hline USA & 0 & 0 & 1 & 13 & 135 & 64 & 127 & 1815 & 2155 \\
\hline Total-out & 3917 & 1726 & 804 & 2206 & 5279 & 9081 & 12328 & 2746 & 38087 \\
\hline
\end{tabular}

Table 7. Traffic flows between high and low-efficiency countries

\begin{tabular}{|l|l|l|l|}
\hline & $\begin{array}{l}\text { Low- } \\
\text { efficiency }\end{array}$ & $\begin{array}{l}\text { High- } \\
\text { efficiency }\end{array}$ & Total-out \\
\hline Low-efficiency & 17369 & 4185 & 21554 \\
& $(45.6 \%)$ & $(11 \%)$ & $(56.6 \%)$ \\
\hline High-efficiency & 3438 & 13095 & 16533 \\
& $(9 \%)$ & $(34.4 \%)$ & $(43.4 \%)$ \\
\hline Total-In & 20807 & 17280 & 38087 \\
& $(54.6 \%)$ & $(45.4 \%)$ & $(100 \%)$ \\
\hline
\end{tabular}

styles of communication. Thus, notes and comments of workers in one country may be difficult to follow for those in another. Second, there may be a tendency to give lower priority to incidents that originated in another country as was seen in the larger queue sizes when more countries were involved. Third, additional overhead is incurred when an incident returns to the home country for closure. Fourth, there may be a favorable "social-network" effect within a country (and unfavorable, across countries) because workers in the same country are more likely to know each other and this fosters a spirit of familiarity.

We recognize that our study is exploratory and has many limitations. First, the dataset does not provide any information about the nature and content of an incident other than its impact. Thus, we are not able to compare the level of difficulty of incidents within the same impact level. Second, there is no information on the context of an incident. Third, we do not have knowledge of the worker skill levels across countries or the methods used for assignment of incidents to them. Fourth, cost of living is only an approximate proxy of wages levels of IT workers in different countries, and accurate estimates of wages can lead to a more precise analysis along the same lines. Finally, we could not control for skill levels across workers and countries to understand how this factor affects the nature of international traffic flows because this data is not available.

\section{Conclusions}

In this paper, we studied the performance of a help desk operation at a large multinational organization using a rich dataset that provides information about each step involved in resolving a problem incident. Our main contribution is to provide a deeper understanding of the the nature of traffic flows across countries, and also to break this down by low- and high-efficiency countries. We found that $80 \%$ of traffic flows were mostly within each of these two groups. Our analysis also shows that resolution of incidents takes longer and is more inefficient when more countries participate in them. The cost of living advantages of low-cost countries such as Poland and India outweighed the efficiency advantages of high-cost countries such as US and Sweden.

For optimal work allocation, we believe that existing algorithms should consider country characteristics, such as resource availability, skill levels and relative resource costs, to build a profile of the workforce of each country that can be used in assigning work to multinational teams along with other factors like quality, priority, compatibility, risk, etc. In addition, it would be useful to build cross-country profiles to see if one pair of countries cooperates better than another one. In any case, an incident should not be scattered across many countries unless necessitated by skill availability because each additional country adds its own layer of complexity.

\section{References}

[1] Arias, M., Rojas, E. BPIC 2013. Volvo Incident and Problem Management Behavior Analysis. Proceedings of the 3rd Business Process Intelligence Challenge, 9th International BPI Workshop (BPI 2013), Beijing, 2013.

[2] Arias, M., Munoz-Gama, J., \& Seplveda, M. (2018). Towards a Taxonomy of Human Resource Allocation Criteria. In E. Teniente \& M. Weidlich (Eds.), BPM Workshops (pp. 475-483). Cham: Springer.

[3] BPI Challenge, 2013. http://www.win.tue.nl/bpi/doku.php?id=2013 
Table 8. Cost of living index in "Top-8" countries (source: numbeo.com)

\begin{tabular}{|c|c|c|c|c|c|c|c|c|}
\hline Country & India & Poland & China & Brazil & Belgium & Sweden & USA & France \\
\hline Cost of Living & 28 & 38 & 42 & 47 & 73 & 79 & 80 & 80 \\
\hline
\end{tabular}

Table 9. Calculation of domestic \%, incidents per worker and monetary cost per incident

\begin{tabular}{|c|c|c|c|c|c|c|c|c|}
\hline Country & Workers & $\begin{array}{c}\text { Total \# of } \\
\text { Incidents }\end{array}$ & $\begin{array}{c}\text { Domestic } \\
\text { Incidents }\end{array}$ & Domestic & $\begin{array}{c}\text { Incidents } \\
\text { Per worker }\end{array}$ & $\begin{array}{c}\text { Events/ } \\
\text { Incident }\end{array}$ & $\begin{array}{c}\text { Cost of } \\
\text { Living }\end{array}$ & $\begin{array}{c}\text { Cost/ } \\
\text { Incident }\end{array}$ \\
\hline India & 243 & 461 & 129 & 27.98 & 1.90 & 8.12 & 28 & 227.36 \\
\hline Poland & 92 & 815 & 128 & 15.71 & 8.86 & 10.46 & 38 & 397.48 \\
\hline China & 40 & 93 & 41 & 44.09 & 2.33 & 7.53 & 42 & 316.26 \\
\hline Brazil & 127 & 225 & 259 & 115.11 & 1.77 & 8.29 & 47 & 389.63 \\
\hline Belgium & 90 & 359 & 54 & 15.04 & 3.99 & 7.89 & 73 & 575.97 \\
\hline Sweden & 581 & 1520 & 960 & 63.16 & 2.62 & 7.77 & 79 & 613.83 \\
\hline USA & 151 & 372 & 217 & 58.33 & 2.46 & 6.68 & 80 & 534.40 \\
\hline France & 131 & 248 & 51 & 20.56 & 1.89 & 8.45 & 80 & 676.00 \\
\hline
\end{tabular}

[4] Cabanillas, C., et al. Priority-based human resource allocation in business processes. International Conference on Service-Oriented Computing. Springer Berlin Heidelberg (2013)

[5] Chatman, J. A., \& Flynn, F. J. (2001). The influence of demographic heterogeneity on the emergence and consequences of cooperative norms in work teams. Academy of Management Jrnl, 44(5), 956-974

[6] R. Conforti, et al. A recommendation system for predicting risks across multiple business process instances. Decision Support Systems 69 (2015): 1-19

[7] Earley, P. C., \& E. Mosakowski. Creating Hybrid Team Cultures: An Empirical Test of Transnational Team Functioning. The Academy of Management Journal, vol. 43, no. 1, 2000, pp. 26-49

[8] Erasmus, J., et al. (2018). A method to enable ability-based human resource allocation in business process management systems. In D. Karagiannis, R. A. Buchmann, \& M. Kirikova (Eds.), The Practice of Enterprise Modeling - 11th IFIP WG 8.1. Working Conference, PoEM 2018, Proceedings (pp. 37-52)

[9] Hambrick, D.C., et al. 1998. When groups consist of multiple nationalities: towards a new understanding of the implications. Organization Studies 19(2): 181-205

[10] Kunze, F., Boehm \& S.A., Bruch, H. (2016) Age diversity, age discrimination climate and performance consequencesa cross organizational study. Journal of organizational behavior 32 (2), 264-290.

[11] Kumar, A., W. van der Aalst \& E. Verbeek. Dynamic work distribution in workflow management systems: How to balance quality and performance. J. Manage. Inf. Syst. Vol. 18, No. 3, Winter 2002, 157-193

[12] Liu, R., Agarwal, S., Sindhgatta, R. R. \& Lee, J. Accelerating collaboration in task assignment using a socially enhanced resource model, BPM 2013: 251-258, Springer (2013)

[13] Marinova, J., Plantenga, J. \& Remary, C. (2016). Gender Diversity and Firm Performance: Evidence form Dutch and Danish boardroom. The International Journal of Human Resource Management, 27(15), 1777-1790

[14] Nielsen, B. B. \& Nielsen, S. (2013). Top management team nationality diversity and firm performance: A multilevel study. Strat. Management Journal, 34, 373-382

[15] Noether G.E. Introduction to Wilcoxon (1945) individual comparisons by ranking methods. In: Kotz S., Johnson N.L. (eds) Breakthroughs in Statistics. Springer Series in Statistics (Perspectives in Statistics). Springer, New York, NY

[16] N. Russell, et al. 2005: Workflow data patterns: identification, representation and tool support. In: Proceedings of the 24th international conference on Conceptual Modeling (ER'05), L. Delcambre, et al. (Eds.). Springer-Verlag, Berlin, Heidelberg, 353-368

[17] Sag, S., Ramazan K. \& Sezen, B.. Factors affecting multinational team performance. Procedia-Social and Behavioral Sciences 235 (2016): 60-69

[18] Schonig, S., Cabanillas, C., Jablonski, S. \& Mendling, J. A framework for efficiently mining the organisational perspective of business processes. Decision Support Systems 89 (2016): 87-97

[19] Scott, W.R. Institutions and Organizations: Ideas and Interests, 3rd ed. Sage Publications, Los Angeles, CA, 2008

[20] M. Song \& W.M.P. van der Aalst. Towards comprehensive support for organizational mining. Decision Support Systems, 46(1) (2008): 300-317

[21] Van der Aalst, W.M.P.: Discovery, Conformance and Enhancement of Business Processes. Springer, 2016

[22] Watson, Warren E., et al. Cultural Diversity's Impact on Interaction Process and Performance: Comparing Homogeneous and Diverse Task Groups. The Academy of Management Jrnl, vol. 36, no. 3, 1993, 590-602

[23] Zoogah, D.B., Vora, D., Richard, O., \& Peng, M.W. (2011). Strategic team alliance diversity, coordination, and effectiveness. The International Journal of Human Resource Management, 22(3), 510-529 